\title{
Éditorial
}

\section{Internet, le palmarès 2007}

Tous les ans, lors d'une réunion du Comité de rédaction, nous analysons les résultats des téléchargements de notre site Internet. Rappelons que notre site www.radioprotection.org est librement consultable et téléchargeable par tous les abonnés et les adhérents à la SFRP. Pour ceux qui ne nous ont pas encore rejoint, le téléchargement est payant pour les trois dernières années, au-delà nos articles sont en libre accès, ce que les professionnels appellent l' «Open Access ».

Alors vous imaginez que la découverte de ce palmarès est pleine d'intérêt pour nous, il nous permet de voir si nos orientations et encouragements correspondent bien aux souhaits de nos lecteurs et membres de la société.

Je ne vous ferai pas attendre plus longtemps : le lauréat 2007 est Jean-Christophe Varin qui, avec P. Casanova, P. Benjamin et M. Rey ont signé l'article «Étude de postes de travail ; le concept et sa traduction en pratique ${ }^{1}$. Parmi les articles uniquement consultables par nos adhérents, viennent ensuite Sébastien Baechler, P. Monnin, A. Aroua, J.F. Valley, M. Perrier, F.R. Verdun de Suisse, pour «Situation actuelle de l'irradiation du patient en radiologie dentaire », et Gonzague Abela pour «Radioprotection et interventions de radiographie industrielle $»^{3}$. Félicitons tous ces auteurs.

Alors, pourquoi sommes-nous satisfaits ?

Certes la médaille d'or est remportée par un membre du Comité de rédaction, ce qui ne gâche rien, mais ce n'est pas la cause principale de notre satisfaction. Non, ce palmarès montre bien que nos lecteurs et adhérents attendent en priorité des articles concrets qui décrivent des situations de radioprotection rencontrées par tout un chacun. Il montre aussi que les présentations à des journées de la SFRP peuvent être transformées en excellents articles et ceci sans compromission, car ces articles, comme tous les articles, sont évalués par des lecteurs anonymes avant toute publication.

Et bien continuons sans oublier, et nous l'avons vu pour le dernier numéro, que ces articles «pratiques» doivent côtoyer dans nos colonnes des articles plus fondamentaux que les lecteurs étrangers consultent. La revue doit être comme notre société : le parfait reflet de notre diversité.

Alors, à vos plumes !

H. Métivier

Président du comité de rédaction

$\begin{array}{ll}1 & \text { Radioprotection (2006) Vol. 41, } \mathrm{N}^{\circ} 2,201-214 . \\ 2 & \text { Radioprotection (2006) Vol. 41, N } \mathrm{N}^{\circ} 3,333-344 . \\ 3 & \text { Radioprotection (2006) Vol. 41, N } \mathrm{N}^{\circ} 2,169-187 .\end{array}$

DOI: 10.1051/radiopro:2008033

RADIOPROTECTION - VOL. 43 - N 3 (2008) 